\title{
IRE-1 Dependent Expression of Phosphoribosyl Pyrophosphate Synthetase Genes in U87 Glioma Cells: Effect of Glucose or Glutamine Deprivation
}

\author{
Dmytro O Minchenko ${ }^{1,2}$, lana A Garmash'1, Yulia M Bashta', Ganna S Kustkova', Yulianna D Zalesna ${ }^{3}$, Andreas Bikfalvi ${ }^{4}$ and Oleksandr H \\ Minchenko ${ }^{1 *}$
}

${ }^{1}$ Department of Molecular Biology, Palladin Institute of Biochemistry, National Academy of Sciences of Ukraine, Kyiv 01601, Ukraine ${ }^{2}$ Departments of Pediatrics, National O.O. Bogomolets Medical University, Kyiv 01601, Ukraine

${ }^{3}$ Department of Neurology and Reflexotherapy, Shupik National Medical Academy of Post-Graduate Education, Kyiv 04112, Ukraine

${ }^{4}$ INSERM U1029, Angiogenesis and Cancer Microenvironment Laboratory, University Bordeaux 1, Talence 33405, France

\begin{abstract}
Nutrient deprivation conditions are capable to induce the endoplasmic reticulum stress and are responsible for regulation of the expression of numerous growth factors which control the cell proliferation and angiogenesis in malignant tumors, including glioma. The ERN1 mediated endoplasmic reticulum stress response-signalling pathway is tightly linked to the proliferation because the blockade of ERN1 function suppresses the tumor growth via specific changes in the cell transcriptome. Activation of the pentose phosphate pathway is an important factor of enhanced cell proliferation and tumor growthbecause it controls the synthesis of nucleotides and respectively nucleic acids. We studied the effect of blockade the ERN1 signaling enzyme function in glioma cells on the expression of phosphoribosyl pyrophosphate synthetase (PRPS), an important enzyme of pentose phosphate pathway, as well as its dependence of glucose and glutamine deprivation.
\end{abstract}

The expression of different genes of PRPS enzyme in glioma cells with complete suppression of ERN1 enzyme activity was measured by qPCR. For glucose and glutamine deprivation conditions the cells were exposurein the glucose or glutamine free medium for $16 \mathrm{hrs}$.

The expression of PRPS1 and PRPS2 genes as well as phosphoribosyl pyrophosphate synthetase associated protein 1(PRPSAP1) is significantly increased in U87 glioma cells with suppressed function of ERN1 gene, but the expression PRPSAP2 gene is decreased. It was also shown that glucose or glutamine deprivation leads to suppression of PRPS1 and PRPS2 gene expressions in U87 glioma cells; however, the blockade of ERN1 function in glioma cells modifies the effect of glutamine deprivation. Moreover, the expression of PRPSAP1 and PRPSAP2 genes was resistant to glucose deprivation condition in control glioma cells, but increased in glioma cells with ERN1 loss of function.

Results of this investigation demonstrated that the blockade of ERN1 signaling enzyme function affects the expression of genes encoding PRPS enzyme subunits in different ways and that dysregulation of the expression of important regulatory subunits of PRPS (PRPSAP1 and PRPSAP2) possibly changes the activity of this enzyme and participates in suppression of glioma growth via pentose phosphate pathway.

Keywords: Gene expression; Pentose phosphate pathway; Phosphoribosyl pyrophosphate synthetase; ERN1; Glucose deprivation; Glioma cells

Abbreviations: PRPS: Phosphoribosyl Pyrophosphate Synthetase; PRPSAP: Phosphoribosyl Pyrophosphate Synthetase Associated Protein; ERN1: Endoplasmic Reticulum-Nuclei-1

\section{Introduction}

Malignant gliomas are highly aggressive tumors and are characterized by marked angiogenesis and extensive tumor cell invasion into the normal brain parenchymaand to date there is no efficient treatment available. Pentose phosphate pathway is necessary for purine metabolism as well as nucleotide biosynthesis and plays an important role in the regulation of proliferative processes and possibly participates in endoplasmic reticulum stress, which is an important factor of tumor growth and many other diseases [1-5]. The endoplasmic reticulum is a key organelle in the cellular response to ischemia, hypoxia, and some chemicals which activate a complex set of signaling pathways named the unfolded protein response. This adaptive response is activated upon the accumulation of misfolded proteins in the endoplasmic reticulum and is mainly mediated by endoplasmic reticulum to nuclei- 1 signaling enzyme (ERN1; also named by inositol requiring enzyme-1alpha,
IRE1). ERN1 is the dominant signaling pathway of the unfolded protein response to the accumulation of misfolded proteins and represents a key regulator of life and death processes [6-9]. As such, it participates in the early cellular response to the accumulation of misfolded proteins in the lumen of the endoplasmic reticulum $[10,11]$.

Two distinct catalytic domains of the bifunctional signaling enzyme ERN1 were identified: a serine/threonine kinase and an endoribonuclease which contribute to ERN1 signalling. The ERN1-

${ }^{*}$ Corresponding author: Oleksandr $\mathrm{H}$ Minchenko, Department of Molecular Biology, Palladin Institute of Biochemistry, National Academy of Sciences of Ukraine, 9 Leontovycha St., Kyiv 01601, Ukraine, Tel: +38-044-235-6151; Fax: +38-044-279-6365; E-mail: ominchenko@yahoo.com

Received June 11, 2013; Accepted July 12, 2013; Published July 17, 2013

Citation: Minchenko DO, Garmash IA, Bashta YM, Kustkova GS, Zalesna YD, et al. (2013) IRE-1 Dependent Expression of Phosphoribosyl Pyrophosphate Synthetase Genes in U87 Glioma Cells: Effect of Glucose or Glutamine Deprivation. Int J Genomic Med 1: 104. doi:10.4172/2332-0672.1000104

Copyright: (c) 2013 Minchenko DO, et al. This is an open-access article distributed under the terms of the Creative Commons Attribution License, which permits unrestricted use, distribution, and reproduction in any medium, provided the original author and source are credited. 
Citation: Minchenko DO, Garmash IA, Bashta YM, Kustkova GS, Zalesna YD, et al. (2013) IRE-1 Dependent Expression of Phosphoribosyl Pyrophosphate Synthetase Genes in U87 Glioma Cells: Effect of Glucose or Glutamine Deprivation. Int J Genomic Med 1: 104. doi:10.4172/2332-0672.1000104

associated kinase activity autophosphorylates and dimerizes this enzyme, leading to the activation of its endoribonuclease domain, degradation of a specific subset of mRNA and initiation of the preXBP1 (X-box binding protein 1) mRNA splicing [12]. Mature XBP1 mRNA splice variant encodes a transcription factor that has different C-terminus amino acid sequence and stimulates the expression of hundreds of the unfolded protein response-specific genes $[9,12,13]$. However, XBP1s has additional functions, especially in the regulation of glucose homeostasis [14].

The endoplasmic reticulum stress is recognized as an important determinant of cancer and contributes to the expression profile of many regulatory genes resulting in proliferation and apoptosis $[3,9,13]$. Moreover, the malignant tumors use the endoplasmic reticulum stress and ischemia for activation of proliferation, because the endoplasmic reticulum stress response-signalling IRE-1 pathway is linked to the apoptosis as well as cell death processes and suppression of IRE-1 gene function significantly decreases the tumor growth $[15,16]$.

The pentose phosphate pathway has an important role in the regulation of glycolysis and proliferation during different stress conditions and participates in control of apoptotic cell death. Phosphoribosyl pyrophosphate synthetase plays a central role in tumor growth because it catalyzes the phosphoribosylation of ribose5-phosphate to 5-phosphoribozyl-1-pyrophosphate which is necessary for purine metabolism and nucleotide biosynthesis [4].

The aim of this study was the investigation of the expression of different phosphoribosyl pyrophosphate synthetase genes in U87 glioma cells with ERN1 deficiency and decreased proliferative activity to find some molecular mechanisms between suppression of ERN1 function, glioma growth from these cells and the activity of pentose phosphate pathway.

\section{Materials and Methods}

The glioma cell line U87 was obtained from ATCC (USA) and grown in high glucose ( $4.5 \mathrm{~g} / \mathrm{l})$ Dulbecco's modified Eagle's minimum essential medium (DMEM; Gibco, Invitrogen, USA) supplemented with glutamine $(2 \mathrm{mM}), 10 \%$ fetal bovine serum (Equitech-Bio, Inc., USA), penicillin (100 units/ml; Gibco) and streptomycin $(0.1 \mathrm{mg} /$ $\mathrm{ml}$; Gibco) at $37^{\circ} \mathrm{C}$ in a $5 \% \mathrm{CO}_{2}$ incubator. In this work we used two sublines of this glioma cell line. One subline was obtained by selection of stable transfected clones with overexpression of pcDNA 3.1 vector, which was used for creation of dominant-negative ERN1 constructs (dnERN1). This untreated subline of glioma cells (control glioma cells) was used as control 1 in the study of glucose and glutamine deprivations on the expression level of PRPS1, PRPS2, PRPSAP1, and PRPSAP2 genes. Second subline was obtained by selection of stable transfected clones with overexpression of dnERN1 and has suppressed both protein kinase and endoribonuclease activities of this bifunctional sensing and signaling enzyme of endoplasmic reticulum stress. These cells were obtained from Prof. M. Moenner (France) [15,16].

The expression level of studied genes in these cells was compared with cells, transfected by vector (control 1), but subline which overexpress dnERN1 was also used as control 2 for investigation the effect of glucose and glutamine deprivation conditions on the expression level of these genes under blockade ERN1 function. For creation of these deprivation conditions we changed the growing medium in culture plates with DMEM without glucose or glutamine and exposed to these conditions for $16 \mathrm{hrs}$.

The enzymatic activity of ERN1 in glioma cells that overexpress
dnERN1 construct was previously analyzed by detection of XBP1 alternative splice variant (XBP1s), a key transcription factor in ERN1 signaling, and the level of ERN1 phosphorylated isoform in cells treated by tunicamycin $(0.01 \mathrm{mg} / \mathrm{ml}$ for 2 hours $)$ [17].

Total RNA was extracted from glioma cells using Trizol reagent according to manufacturer protocol (Invitrogen, USA). Cells were extracted with $2 \mathrm{ml}$ of Trizol reagent and $0.4 \mathrm{ml}$ of chloroform was added to cell lysate, mix and spin down. RNA was precipitated with an equal volume of 2-propanol. RNA pellets were washed with $75 \%$ ethanol and dissolved in nuclease-free water. For additional purification the RNA samples were re-precipitated with ethanol and dissolved again in nuclease-free water.

Quani Tect Reverse Transcription Kit (QIAGEN, Germany) was used for cDNA synthesis according to manufacturer protocol. For this aim $1 \mu \mathrm{g}$ of RNA was incubated for 2 min at $42^{\circ} \mathrm{C}$ with gDNA Wipeout buffer, and then placed immediately on ice. Add reverse-transcription master mix, containing Quantiscript Reverse Transcriptase, Quantiscript RT Buffer and RT Primer Mix, incubated for $15 \mathrm{~min}$ at $42^{\circ} \mathrm{C}$ for synthesis of cDNA, and then incubated for $3 \mathrm{~min}$ at $95^{\circ} \mathrm{C}$ to inactivate transcriptase.

The expression level of PRPS1, PRPS2, PRPSAP1, and PRPSAP2mRNA were measured in glioma cell line U87 and its subline (clone 1C5) by real-time quantitative polymerase chain reaction using "Mx 3000PQPCR" (Stratagene,USA) and Absolute qPCR SYBRGreenMix (Thermo Fisher Scientific, ABgene House, UK). For qPCR analysis initial step was incubationfor $10 \mathrm{~min}$ at $95^{\circ} \mathrm{C}$, and then 40 cycles included incubation for $0.5 \mathrm{~min}$ at $95^{\circ} \mathrm{C}$, for $0.5 \mathrm{~min}$ at $55^{\circ} \mathrm{C}$ and for $0.5 \mathrm{~min}$ at $72^{\circ} \mathrm{C}$. At the end of qPCR was final step for detection of dissociation curve. Polymerase chain reaction was performed in triplicate.

For amplification of phosphoribosyl pyrophosphate synthetase 1 (PRPS1) cDNA we used next primers: forward (5'-AAGAACGGAAGAAGGCCAAT-3' and reverse (5'-GACCGGAGAAGATTCCATGA-3') primers. The nucleotide sequences of these primers correspond to sequences 786-805 and 971952 of human PRPS1 cDNA (GenBank accession number NM_002764). The size of amplified fragment is $186 \mathrm{bp}$.

Two other primers were used for real time RCR analysis of phosphoribosyl pyrophosphate synthetase 2 (PRPS2) cDNA forward (5'-AAACATTGCCGAGTGGAAG-3' and reverse (5'-TGACGACAACAGCCTCAAAG-3') primers. The nucleotide sequences of these primers correspond to sequences 613-632 and 957948 of human PRPS2 cDNA (GenBank accession number NM_002765). The size of amplified fragment is $345 \mathrm{bp}$.

For amplification of phosphoribosyl pyrophosphate synthetaseassociated protein 1 (PRPSAP1) cDNA we used next primers: forward 5'-GTCCTATGCGGAGAGACTGC-3' and reverse 5'- TAGGCGCCTCTCTCTTTCAG-3'. These oligonucleotides correspond to sequences 1088-1107 and 1366-1347 of human PRPSAP1 cDNA (GenBank accession number NM_002766). The size of amplified fragment is $279 \mathrm{bp}$.

For amplification of phosphoribosyl pyrophosphate synthetaseassociated protein 2 (PRPSAP2) cDNA we used next primers: forward 5'-CACCCATGGTCAGAAGTGTG-3' and reverse 5'- TTAATCTTGGGGCACTGGAG -3'. These oligonucleotides correspond to sequences 698-717 and 980-961 of human PRPSAP2 
Citation: Minchenko DO, Garmash IA, Bashta YM, Kustkova GS, Zalesna YD, et al. (2013) IRE-1 Dependent Expression of Phosphoribosyl Pyrophosphate Synthetase Genes in U87 Glioma Cells: Effect of Glucose or Glutamine Deprivation. Int J Genomic Med 1: 104. doi:10.4172/2332-0672.1000104

Page 3 of 5

cDNA (GenBank accession number NM_002767). The size of amplified fragment is $328 \mathrm{bp}$.

The amplification of beta-actin (ACTB) cDNA was performed using forward-5'- GGACTTCGAGCAAGAGATGG-3' and reverse5'AGCACTGTGTTGGCGTACAG-3' primers. These primers nucleotide sequences correspond to 747-766 and 980-961 of human ACTB cDNA (GenBank accession number NM_001101). The size of amplified fragment is $234 \mathrm{bp}$. The expression of beta-actin mRNA was used as control of analyzed RNA quantity. The primers were received from "Sigma" (USA).

An analysis of quantitative PCR was performed using special computer program "Differential expression calculator" and statistical analysis-in Excel program. The values of PRPS1, PRPS2, PRPSAP1, and PRPSAP2mRNA expressions were normalized to the expression of beta-actin mRNA and represent as percent of control (100\%). All values are expressed as mean \pm SEM from triplicate measurements performed in 4 independent experiments. The statistical significance of differences was calculated by Students t-test. $\mathrm{p}<0.05$ was considered to be a significant difference.

\section{Results}

As shown in Figure 1, the expression level of phosphoribosyl pyrophosphate synthase genes PRPS1, PRPS2, and PRPSAP1 significantly increasedin glioma U87 cells with knockdown of ERN1, a major sensor and signaling enzyme of endoplasmic reticulum stress, being more significant for PRPS1 gene. At the same time, the expression of PRPSAP2 gene is decreased in these glioma cells.

It was also shown that exposure of U87 glioma cells under glucose or glutamine deprivation conditions led to suppression of PRPS1 gene expression both in control glioma cells and cells with ERN1 knockdown, but more strong effect of glutamine deprivation condition was observed in glioma cells without ERN1 function (Figure 2). As shown in Figure 3 , the similar changes were observed in the expression level of PRPS2 gene in control glioma cells as well as in cells with suppressed function of ERN1. For this gene was also shown more strong effect of glutamine deprivation condition in glioma cells with ERN1 knockdown.

At the same time, the expression of PRPSAP1 gene was resistant

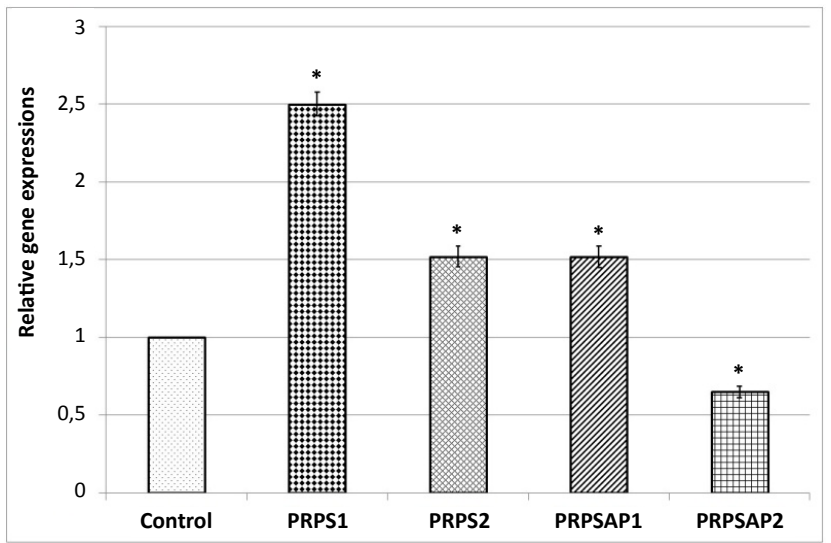

Figure 1: The expression of PRPS1, PRPS2, PRPSAP1, and PRPSAP2 mRNA in glioma cell line U87, transfected with vector, and its subline with a deficiency of the signaling enzyme ERN1 (dnERN1) measured by qPCR. Values of PRPS1, PRPS2, PRPSAP1, and PRPSAP2mRNA expressions were normalized to beta-actin mRNA expression and represent as percent of control $(100 \%) ; n=4 ;{ }^{*}-\mathrm{P}<0.05$ as compared to control $1 ;{ }^{* *}-\mathrm{P}<0.05$ as compared to control 2 . to glucose and glutamine deprivation conditions in control glioma cells but suppression of ERN1 signaling enzyme function is modified the sensitivity of this gene expression to both glucose and glutamine deprivation conditions (Figure 4). Thus, the expression of PRPSAP1 gene in glioma cells with ERN1 loss of function is increased in glucose deprivation condition and decreased in glutamine deprivation condition. It was also shown that PRPSAP2 gene expression in control glioma cells was suppressed in glutamine deprivation condition but resistant to glucose deprivation condition (Figure 5). However, in glioma cells withERN1 knockdown the expression of PRPSAP2 was increased both in glucose and glutamine deprivation conditions.

\section{Discussion}

We have studied the expression of four different genes of one enzyme, phosphoribosyl pyrophosphate synthase, encoding two catalytic and two regulatory subunits of associated proteins, in U87 glioma cells with ERN1 deficiency and decreased proliferative activity

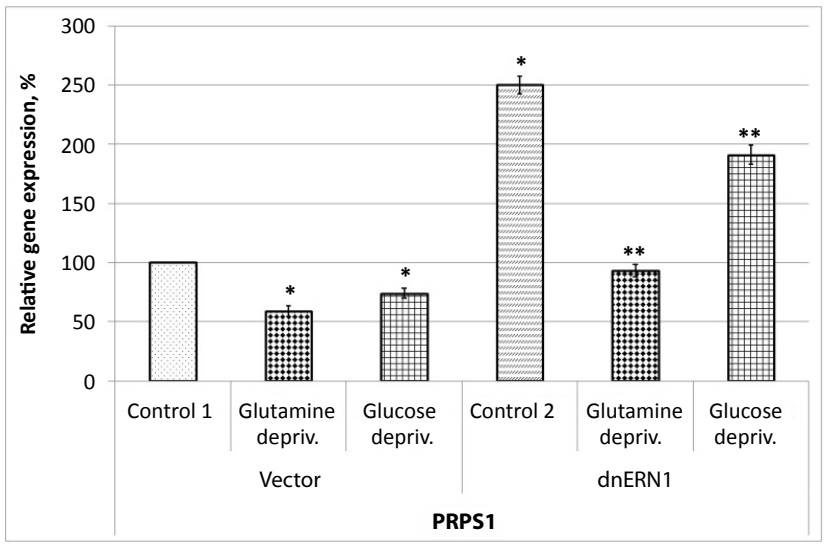

Figure 2: The expression of phosphoribosyl pyrophosphate synthetase 1 (PRPS1) mRNA in glioma cell line U87 (Vector) and its subline with a deficiency of the signaling enzyme ERN1 (dnERN1):effect of glucose or glutamine deprivations. Values of PRPS1 mRNA expressions were normalized to betaactin mRNA expression and represent as percent of control (100 \%);n=4 ${ }^{*}-\mathrm{P}<0.05$ as compared to control 1 ; ${ }^{*}-\mathrm{P}<0.05$ as compared to control 2 .

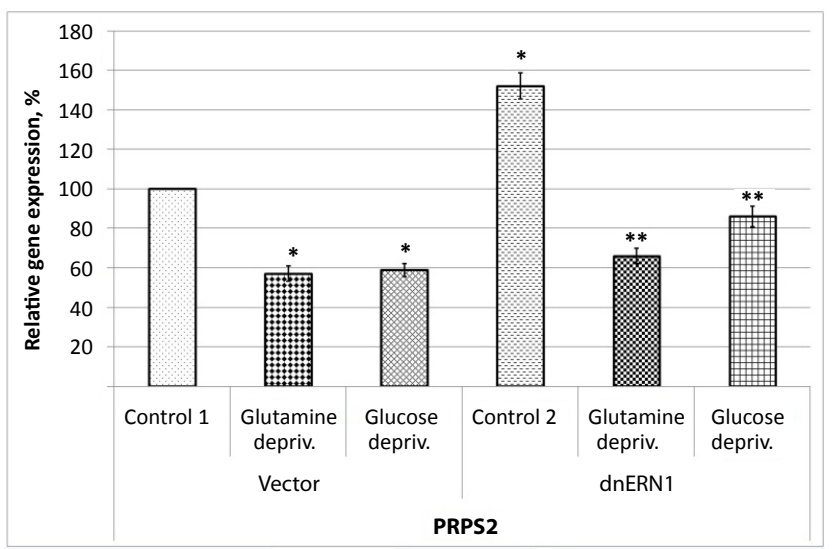

Figure 3: Effect of glucose or glutamine deprivations on the expression of phosphoribosyl pyrophosphate synthetase 2 (PRPS2) mRNA in glioma cell line U87 (Vector) and its subline with a deficiency of the signaling enzyme ERN1 (dnERN1) measured by qPCR. Values of PRPS2 mRNA expressions were normalized to beta-actin mRNA expression and represent as percent of control $(100 \%) ; n=4 ;^{*}-\mathrm{P}<0.05$ as compared to control $1{ }^{* *}-\mathrm{P}<0.05$ as compared to control 2 . 
Citation: Minchenko DO, Garmash IA, Bashta YM, Kustkova GS, Zalesna YD, et al. (2013) IRE-1 Dependent Expression of Phosphoribosyl Pyrophosphate Synthetase Genes in U87 Glioma Cells: Effect of Glucose or Glutamine Deprivation. Int J Genomic Med 1: 104. doi:10.4172/2332-0672.1000104

Page 4 of 5

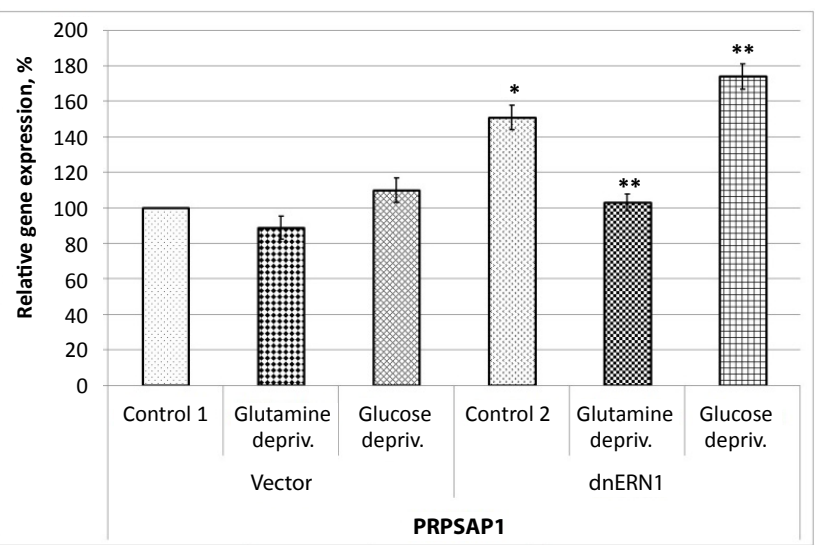

Figure 4: The expression of phosphoribosyl pyrophosphate synthetase associated protein 1 (PRPSAP1) mRNA in glioma cell line U87 (Vector) and its subline with a deficiency of the signaling enzyme ERN1 (dnERN1): effect of glucose or glutamine deprivations. Values of PRPSAP1 mRNA expressions were normalized to beta-actin mRNA expression and represent as percent of control $(100 \%) ; n=4 ;{ }^{*}-\mathrm{P}<0.05$ as compared to control $1 ;{ }^{* *}-\mathrm{P}<0.05$ as compared to control 2 .

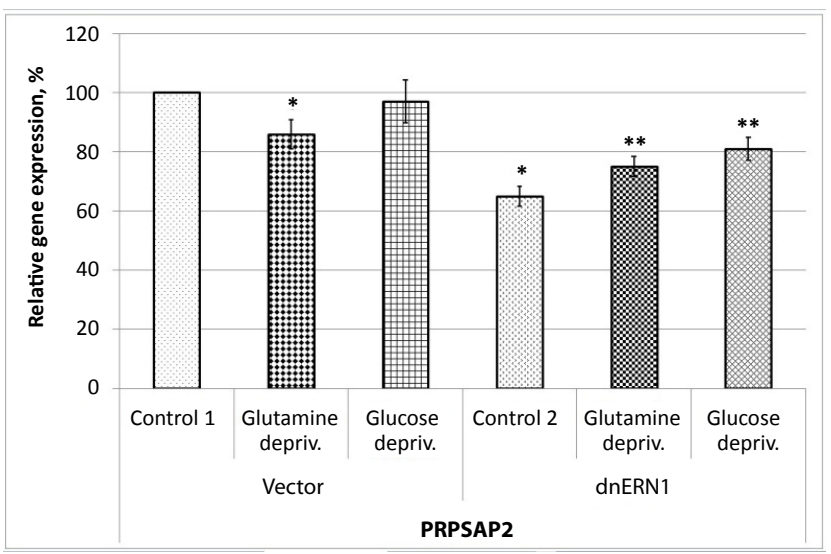

Figure 5: Effect of glucose or glutamine deprivations on the expression of phosphoribosyl pyrophosphate synthetase associated protein 2 (PRPSAP2) mRNA in glioma cell line U87 (Vector) and its subline with a deficiency of the signaling enzyme ERN1 (dnERN1). Values of PRPSAP2 mRNA expressions were normalized to beta-actin mRNA expression and represent as percent of control $(100 \%) ; n=4 ;{ }^{*}-\mathrm{P}<0.05$ as compared to control $1 ;{ }^{* *}-\mathrm{P}<0.05$ as compared to control 2 .

with the goal to identify some molecular mechanisms between suppression of ERN1 function, glioma growth from these cells and the activity of pentose phosphate pathway. However, little is known about the molecular mechanisms of the regulation of different PRPS genes, in particular, its participation in ERN1 signalling pathway of endoplasmic reticulum stress $[5,18]$. Results of this investigation demonstrate that expression of different subunits of phosphoribosyl pyrophosphate synthase in glioma cells significantly depends from endoplasmic reticulum stress, in particular, its ERN1signalling system and thatthe blockade of ERN1 enzyme leads to dysregulation of the expression of different subunits of PRPS. Thus, in glioma cells with ERN1 knockdown we have shown an induction of PRPS1, PRPS2, and PRPSAP1 gene expressions and suppression of PRPSAP2 gene, but more significant changes was founded for PRPS1 gene. It is interested to note that these changes in the expression of PRPS1 and PRPS2 genes in ERN1 knockdown glioma cells are correlated with down regulation of these gene expressions in control glioma cells under glutamine and glucose deprivation conditions which induce the endoplasmic reticulum stress.

Thus, the blockade of ERN1 gene function in glioma cells leads to dysregulation of the expression of different subunits of phosphoribosyl pyrophosphate synthase and possibly decreases the level of 5-phosphoribosyl-1-pyrophosphate, which is necessary for purine metabolism and nucleotide biosynthesis, and participates in strong decrease of glioma growth through suppression of nucleic acid synthesis and the level of GTP, ATP and NAD [19]. Moreover, direct role of nucleotide metabolism was shown in C-MYC-dependent proliferation of melanoma cells via interactions with PRPS2 and other genes [20]. Thus, all genes encoding phosphoribosyl pyrophosphate synthetase are responsible from endoplasmic reticulum stress, in particular its ERN1 signalling pathway. However, this response is different for different subunits of PRPS enzyme.

Moreover, it is known that the endoplasmic reticulum stress response ERN1 signalling pathway is linked to the apoptosis as well as proliferation processes because malignant tumors use the endoplasmic reticulum stress and ischemia for activation of proliferation processes and suppression of ERN1 gene function significantly decreases the glioma growth $[3,15,16]$. Thus, our findings concerning dysregulation of different PRPS gene expressions after blockade of ERN1 signalling enzyme function in glioma cells possibly have relation to suppression of glioma growth from these cells.

Moreover, we have shown that the glucose deprivation condition also affects the expression of PRPS1 and PRPS2 genes in control as well as ERN1 knockdown U87 glioma cells and that this effect of glucose deprivation on the expression of both PRPS1 and PRPS2 gene was similar. At the same time, the expression of PRPS associated protein genes (PRPSAP1 and PRPSAP2) is resistant to glucose deprivation condition in control glioma cells but is increased in glioma cells with ERN1 loss of function. Thus, the glutamine deprivation condition affects the expression of different phosphoribosyl pyrophosphate synthase genes except PRPSAP1 in control glioma cells; however, ERN1 knockdown significantly modifies the effect of glutamine deprivation on the expression of these phosphoribosyl pyrophosphate synthase genes. At the same time, the exposure cells to glutamine deprivation condition leads to suppression of PRPSAP1 gene expression only in U87 glioma cells without ERN1 enzyme function.

Moreover, the blockade of ERN1 gene function leads to upregulation of PRPSAP2 gene expression in U87 glioma cells under glutamine deprivation condition. This data conforms to downregulation of this gene expression in glioma cells without ERN1 enzyme function. Thus, the effect of glutamine deprivation condition on the expression of different phosphoribosyl pyrophosphate synthase genes in U87 glioma cells depends from ERN1 function. These results are consistent with data of other investigation where was shown the dependence of different gene expressions under nutrient deprivation condition as well as hypoxia from ERN1 signaling enzyme function [17,19-22]. There are different effects of ERN1 blockade on hypoxic or nutrient deprivation regulations of gene expression, enhance, reduce or eliminate these regulations.

Results of this investigation clearly demonstrated that the blockade of ERN1 signaling enzyme function affects the expression ofgenes encoding PRPS enzyme subunits in different ways and that dysregulation of the important regulatory subunits of PRPS (PRPSAP1 and PRPSAP2) possibly participates in suppression of glioma growth also via pentose phosphate pathway. It is possible that 
Citation: Minchenko DO, Garmash IA, Bashta YM, Kustkova GS, Zalesna YD, et al. (2013) IRE-1 Dependent Expression of Phosphoribosyl Pyrophosphate Synthetase Genes in U87 Glioma Cells: Effect of Glucose or Glutamine Deprivation. Int J Genomic Med 1: 104. doi:10.4172/2332-0672.1000104

Page 5 of 5

these important regulatory subunits of PRPS enzyme could be used as targets for developing of new approaches for suppression of glioma growth. However, the detailed molecular mechanisms for PRPS gene expressions under endoplasmic reticulum stress condition are complex and the biological significance remains to be determined.

\section{References}

1. Zhang K, Kaufman RJ (2004) Signaling the unfolded protein response from the endoplasmic reticulum. J Biol Chem 279: 25935-25938.

2. Marciniak SJ, Ron D (2006) Endoplasmic reticulum stress signaling in disease. Physiol Rev 86: 1133-1149.

3. Moenner M, Pluquet O, Bouchecareilh M, Chevet E (2007) Integrated endoplasmic reticulum stress responses in cancer. Cancer Res 67: 10631-10634.

4. Mannava S, Grachtchouk V, Wheeler LJ, Im M, Zhuang D, et al. (2008) Direct role of nucleotide metabolism in C-MYC-dependent proliferation of melanoma cells. Cell Cycle 7: 2392-2400.

5. de Brouwer AP, van Bokhoven H, Nabuurs SB, Arts WF, Christodoulou J, et al (2010) PRPS1 mutations: four distinct syndromes and potential treatment. Am J Hum Genet 86: 506-518.

6. Bi M, Naczki C, Koritzinsky M, Fels D, Blais J, et al. (2005) ER stress-regulated translation increases tolerance to extreme hypoxia and promotes tumor growth. EMBO J 24: 3470-3481.

7. Fels DR, Koumenis C (2006) The PERK/elF2alpha/ATF4 module of the UPR in hypoxia resistance and tumor growth. Cancer Biol Ther 5: 723-728.

8. Romero-Ramirez L, Cao H, Nelson D, Hammond E, Lee AH, et al. (2004) XBP1 is essential for survival under hypoxic conditions and is required for tumor growth. Cancer Res 64: 5943-5947.

9. Acosta-Alvear D, Zhou Y, Blais A, Tsikitis M, Lents NH, et al. (2007) XBP1 controls diverse cell type- and condition-specific transcriptional regulatory networks. Mol Cell 27: 53-66.

10. Schröder M (2008) Endoplasmic reticulum stress responses. Cell Mol Life Sc 65: 862-894.

11. Lin JH, Walter P, Yen TS (2008) Endoplasmic reticulum stress in disease pathogenesis. Annu Rev Pathol 3: 399-425.
12. Hollien J, Lin JH, Li H, Stevens N, Walter P, et al. (2009) Regulated Ire1-dependent decay of messenger RNAs in mammalian cells. J Cell Biol 186: 323-331.

13. Aragón T, van Anken E, Pincus D, Serafimova IM, Korennykh AV, et al. (2009) Messenger RNA targeting to endoplasmic reticulum stress signalling sites. Nature 457: 736-740.

14. Lee SK, Kim YS (2013) Phosphorylation of elF2Ît attenuates statin-induced apoptosis by inhibiting the stabilization and translocation of p53 to the mitochondria. Int J Oncol 42: 810-816.

15. Drogat B, Auguste P, Nguyen DT, Bouchecareilh M, Pineau R, et al. (2007) IRE1 signaling is essential for ischemia-induced vascular endothelial growth factor-A expression and contributes to angiogenesis and tumor growth in vivo. Cancer Res 67: 6700-6707.

16. Auf G, Jabouille A, Guérit S, Pineau R, Delugin M, et al. (2010) Inositolrequiring enzyme 1alpha is a key regulator of angiogenesis and invasion in malignant glioma. Proc Natl Acad Sci U S A 107: 15553-15558.

17. Minchenko D, Kubajchuk, Ratushna OO, Komisarenko SV, Minchenko OH (2012) The effect of hypoxia and ischemic conditionon the expression of VEGF genes in glioma U87 cells is dependent from ERN1 knockdown. Adv Biol Chem 2: $198-206$.

18. Yamada Y, Yamada K, Nomura N,Yamano A, Kimura R, et al. (2010) Molecular analysis of two enzyme genes, HPRT1 and PRPS1, causing X-linked inborn errors of purine metabolism. Nucleosides Nucleotides Nucleic Acids 29: 291-294.

19. Drogat B, Bouchecareilh M, North S, Petibois C, Déléris G, et al. (2007) Acute L-glutamine deprivation compromises VEGF-a upregulation in A549/8 human carcinoma cells. J Cell Physiol 212: 463-472.

20. Minchenko DO, Karbovskyi LL, Danilovskyi SV, Kharkova AP, Minchenko OH (2012) Expression of casein kinase genes in glioma cell line U87: effect of hypoxia and glucose or glutamine deprivation. Nat Sci 4: 38-46.

21. Minchenko DO, Karbovskyi LL, Danilovskyi SV, Moenner M, Minchenko OH (2012) Effect of hypoxia and glutamine or glucose deprivation on the expression of retinoblastoma and retinoblastoma-related genes in ERN1 knockdown glioma U87 cell line. Am J Mol Biol 2: 21-31.

22. Minchenko DO, Kharkova AP, Hubenia OV, Minchenko OH (2013) Insulin receptor, IRS1, IRS2, INSIG1, INSIG2, RRA D, and BAIAP2 gene expressions in glioma U87 cells with ERN1 loss of function: effect of hypoxia and glutamine or glucose deprivation. Endocr Regul 47: 15-26. 